\title{
Article \\ Study on Implicit-Type Fractional Coupled System with Integral Boundary Conditions
}

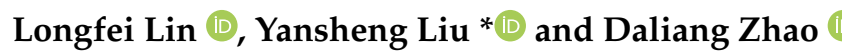 \\ School of Mathematics and Statistics, Shandong Normal University, Jinan 250014, China; \\ 2019020438@stu.sdnu.edu.cn (L.L.); dlzhao928@sdnu.edu.cn (D.Z.) \\ * Correspondence: ysliu@sdnu.edu.cn
}

\begin{abstract}
This paper is concerned with a class of implicit-type coupled system with integral boundary conditions involving Caputo fractional derivatives. First, the existence result of solutions for the considered system is obtained by means of topological degree theory. Next, Ulam-Hyers stability and generalized Ulam-Hyers stability are studied under some suitable assumptions. Finally, one example is worked out to illustrate the main results.
\end{abstract}

Keywords: integral boundary conditions; fractional differential equations; Ulam-Hyers stability; implicit type

\section{Introduction}

This paper aims to establish the sufficient conditions for the existence and stability results of solutions to a class of coupled systems of implicit-type fractional differential equations (IFDEs) involving Caputo fractional derivatives under the integral-type boundary conditions

Citation: Lin, L.; Liu, Y.; Zhao, D. Study on Implicit-Type Fractional Coupled System with Integral Boundary Conditions. Mathematics 2021, 9, 300. https://doi.org/10.3390/ math 9040300

Academic Editor: Christopher Goodrich Received: 30 December 2020 Accepted: 30 January 2021 Published: 3 February 2021

Publisher's Note: MDPI stays neutral with regard to jurisdictional clai$\mathrm{ms}$ in published maps and institutional affiliations.

Copyright: (C) 2021 by the authors. Licensee MDPI, Basel, Switzerland. This article is an open access article distributed under the terms and conditions of the Creative Commons Attribution (CC BY) license (https:// creativecommons.org/licenses/by/ $4.0 /)$.

where $\alpha, \beta \in(1,2], \mathcal{D}$ denotes the standard Caputo fractional derivative; $f_{1}, f_{2} \in C([0,1] \times$ $\mathbb{R} \times \mathbb{R}, \mathbb{R}), h_{1}, h_{2} \in C(C[0,1], \mathbb{R})$, and $g_{1}, g_{2} \in L^{1}[0,1]$.

It is well known that the subject of fractional differential equations (FDEs) has gained considerable popularity and importance due to its wide range of applications in describing the real word problems. FDEs are powerful tools to model the many phenomena of different fields of scientific disciplines and engineering. The study of FDEs is found to be of great value and interest in view of the occurrence of such systems in a variety of problems of applied nature. For example, there are the Lorenz system [1], chaotic synchronization system [2], Chua circuit [3], impulsive problems [4,5], quantum theory [6], stability and controllability problems [7,8], diffusion phenomenon [9-12], and systems of thermoelasticity $[13,14]$, etc. Compared with the classical integral order derivatives, the fractional derivatives provide significant tools for the analysis of memory and hereditary properties. In consequence, many meaningful results in these fields have been obtained. For more details, please see Kilbas et al. [15], Lakshmikantham et al. [16], Podlubny [17], Samko et al. [18], and references therein.

Another topic which has increasingly received interest in recent years is stability theory, which plays an important role in optimization and numerical point of view. In order to deal with the difficulties that arise from exponential Mittag-Leffler and Lyapunovy stability theory, Ulam [19] introduced another kind of stability, that is Ulam-Hyers stability, which was further studied by Hyers [20] in the subsequent years. Since then, many 
achievements in various kinds of differential equations with integer order in this field have been investigated ([21,22], etc.). Nowadays, researchers are focusing on the study of Ulam stability for FDEs-for more details about Ulam stability with FDEs, please see [23-26] and the references therein.

Integral boundary value conditions (IBCs, for short) of various types of differential equations have also been regarded as an important research field in recent years. IBCs play an important role in the study of computational fluid dynamics studies related to blood flow problems. In order to solve the unreasonable problem that the cross-section of blood vessels is always assumed to be circular, IBCs provide an effective and applicable approach. In addition, IBCs also have an important applications in the dynamic behavior of bioprocesses, such as HIV infection. More details can be found in $[27,28]$. Additionally, IBCs have useful applications in regularizing ill-posed parabolic backward problems in time partial differential equations-see, for example, mathematical models for bacterial self-regularization [29]. Some recent investigations on coupled systems of FDEs with IBCs can be found in $[23,27,30-33]$ and the references cited therein.

As a branch of the research on FDEs, the IFDEs have been given widespread attention, due to the fact that many of the problems around managerial and economics are modeled by IFDEs. For more details on IFDEs, one can see [34-36], and references therein. Recently, IFDEs have been properly investigated by many researchers, such as Z. Ali et al. [25], M. Benchohra et al. [24], J. J. Nieto et al. [37], and Samina et al. [38]. They focus on the existence and stability theory. For example, J. J. Nieto et al., in [37], investigated the following implicit fractional differential equations via the Liouville-Caputo derivative:

$$
\left\{\begin{array}{l}
{ }^{c} \mathcal{D}^{\alpha} y(t)=f\left(t, y(t),{ }^{c} \mathcal{D}^{\alpha} y(t)\right), t \in[0, b] \\
y(0)=y_{0}
\end{array}\right.
$$

where $\alpha \in(0,1), f:[0, b] \times \mathbb{R} \times \mathbb{R} \rightarrow \mathbb{R}$ is a continuous function. The existence and uniqueness results are obtained by means of fixed point theory and an approximation method In [38], Samina et al. studied the following IFDEs with antiperiodic boundary conditions involving Caputo fractional derivatives

$$
\left\{\begin{array}{l}
\mathcal{D}^{\alpha} x(t)=\vartheta\left(t, y(t), \mathcal{D}^{\alpha} x(t)\right), \alpha \in(1,2], t \in J \\
\mathcal{D}^{\beta} y(t)=\theta\left(t, x(t), \mathcal{D}^{\beta} y(t)\right), \beta \in(1,2], t \in J \\
x(0)=-x(\eta), x^{\prime}(0)=-x^{\prime}(\eta) \\
y(0)=-y(\eta), y^{\prime}(0)=-y^{\prime}(\eta)
\end{array}\right.
$$

where $\eta \in(0,+\infty), J=[0, \eta]$, and $\vartheta, \theta: J \times \mathbb{R} \times \mathbb{R} \rightarrow \mathbb{R}$ are nonlinear continuous functions. By applying Banach and Schauder's fixed point theorem, the existence and uniqueness results for the considered problem were obtained under some suitable assumptions.

From all of the above works, we find the fact that their nonlinear terms need to satisfy the Lipschitz condition for two variables. The implicit of the corresponding problem can be overcome by this condition, which plays a key role in obtaining the existence results of solutions. Although this condition is convenient, it is very restrictive and excludes a lot of nonlinear IFDEs. Therefore, we want to investigate whether it works when one "certain variable" satisfies the Lipschitz condition in nonlinear terms.

As far as we know, there are few paper studied Ulam-Hyers stability for IFDEs and there is no paper considering such a problem for (1). Thus, the purpose of the present paper is to fill this gap. Moreover, our nonlinear terms only need to satisfy the Lipschitz condition for one "certain variable", which relax the restriction of the nonlinear terms in the literatures $[24,38]$.

We subdivide this paper into the following Sections. Section 2 contains some preliminaries. In Section 3, some transformations are introduced to convert the problem (1) into the corresponding explicit form and integral form. In Section 4, the existence results of solutions will be given and proved by topological degree theory together with the properties of Green function. In Section 5, we establish Ulam-Hyers stability and generalized 
Ulam-Hyers stability under some suitable assumptions. Finally, in Section 6, one example is worked out to support our main results.

\section{Preliminaries}

In this section, we recall some basic definitions and fundamental results necessary for this work throughout this paper. First, we provide some fundamental material about fractional calculus.

Definition 1 ([17]). The Riemann-Liouville standard fractional integral of order $\alpha>0$ of a continuous function $u:(0,+\infty) \rightarrow \mathbb{R}$ is given by

$$
\mathcal{I}^{\alpha} u(t)=\frac{1}{\Gamma(\alpha)} \int_{0}^{t}(t-s)^{\alpha-1} u(s) d s,
$$

provided that the right side integral is pointwise defined on $\mathbb{R}^{+}=:(0,+\infty)$.

Definition 2 ([17]). The Caputo fractional derivative of order $\alpha>0$ of a continuous function $u:(0,+\infty) \rightarrow \mathbb{R}$ is given by

$$
\mathcal{D}^{\alpha} u(t)=\frac{1}{\Gamma(n-\alpha)} \int_{0}^{t} \frac{u^{(n)}(s)}{(t-s)^{\alpha-n+1}} d s,
$$

where $n=[\alpha]+1,[\alpha]$ denotes the integer part of the real number $\alpha$, and provided that the right side integral is pointwise defined on $[0,+\infty)$.

Lemma 1 ([17]). Let $n-1<\alpha<n(n \in \mathbb{N})$. Then,

$$
\mathcal{I}^{\alpha} \mathcal{D}^{\alpha} u(t)=u(t)+c_{0}+c_{1} t+c_{2} t^{2}+\ldots+c_{n-1} t^{n-1},
$$

for some $c_{i} \in \mathbb{R}, i=0,1,2, \ldots, n-1, n=[\alpha]+1$.

Next, we list some basic definitions and fundamental results about topological degree theory. For this sake, let $E$ be a Banach space with norm $\|\cdot\|, D \subset E$, and the operator $\mathcal{A}: D \rightarrow E . \chi(\cdot)$ represents the Kuratowski measure of noncompactness.

Definition 3 ([39]). Assume that $\mathcal{A}$ is continuous and bounded.

(1) If there is a constant $\sigma \geq 0$, such that $\chi(\mathcal{A}(S)) \leq \sigma \chi(S)$ for any bounded $S \subset E$, then $\mathcal{A}$ is called a $\sigma$-set contraction; a $\sigma$-set contraction with $\sigma<1$ is called a strict set contraction.

(2) $\mathcal{A}$ is said to be condensing if $\chi(\mathcal{A}(S)) \leq \chi(S)$ for any bounded $S \subset E$, which is not relatively compact.

Proposition 1 ([39]). If $\mathcal{A}$ is $\chi$-Lipschitz having constants $h$, namely

$$
\|\mathcal{A}(x)-\mathcal{A}(y)\| \leq h\|x-y\|, \quad \forall x, y \in D .
$$

Then $\mathcal{A}$ is h-set contraction in $D$.

Proposition 2 ([39]). If $\mathcal{A}$ is compact, then $\mathcal{A}$ is 0 -set contraction.

Proposition 3 ([39]). If $\mathcal{A}_{1}: D \rightarrow E$ is $\sigma_{1}$-set contraction and $\mathcal{A}_{2}: D \rightarrow E$ is $\sigma_{2}$-set contraction, then $\mathcal{A}$ is $\left(\sigma_{1}+\sigma_{2}\right)$-set contraction.

Theorem 1 ([39]). Let $\mathcal{T}: E \rightarrow E$ be condensing and

$$
\Omega=\{x \in E: \exists 0 \leq \xi \leq 1 \text { with } x=\xi \mathcal{T}(x)\} .
$$


If $\Omega$ is a bounded set in $E$, then $\mathcal{T}$ has at least one fixed point.

\section{Conversion of the Considered System}

In this section, we convert problem (1) into explicit form to overcome the difficulties that arise from implicity. $\mathcal{X}=C[0,1]$ represents a Banach space with norm $\|x\|=\max \{|x(t)|: t \in[0,1]\}$. The product $\mathcal{X} \times \mathcal{X}$ is a Banach space with norm $\|(x, y)\|=\|x\|+\|y\|$. For simplicity and convenience, set

$$
\begin{aligned}
& \delta_{1}=1-\int_{0}^{1} g_{1}(\tau) d \tau, \quad \delta_{2}=1-\int_{0}^{1} \tau g_{1}(\tau) d \tau, \quad \delta=\frac{\left|\delta_{2}\right|}{\left|\delta_{1}\right|+\left|\delta_{2}\right|} \\
& \gamma_{1}=1-\int_{0}^{1} g_{2}(\tau) d \tau, \quad \gamma_{2}=1-\int_{0}^{1} \tau g_{2}(\tau) d \tau, \quad \gamma=\frac{\left|\gamma_{2}\right|}{\left|\gamma_{1}\right|+\left|\gamma_{2}\right|} .
\end{aligned}
$$

Now let us list the following assumptions satisfied throughout the paper.

Hypothesis 1. $f_{1}, f_{2} \in C([0,1] \times \mathbb{R} \times \mathbb{R}, \mathbb{R}), h_{1}, h_{2} \in C(\mathcal{X}, \mathbb{R}), g_{1}, g_{2} \in L^{1}[0,1]$, and $\delta_{2}, \gamma_{2} \neq$ 0 .

Hypothesis 2. There exist constants $k_{1} \in[0, \delta)$ and $k_{2} \in[0, \gamma)$ such that

$$
\begin{aligned}
& \left|h_{1}(x)-h_{1}\left(x_{0}\right)\right| \leq k_{1}\left|x-x_{0}\right|, \quad \forall x, x_{0} \in \mathcal{X} \\
& \left|h_{2}(y)-h_{2}\left(y_{0}\right)\right| \leq k_{2}\left|y-y_{0}\right|, \quad \forall y, y_{0} \in \mathcal{X} .
\end{aligned}
$$

Hypothesis 3. There exist constants $\sigma_{1}, \sigma_{2} \in(0,1)$, such that, for each $u, u_{0}, v, v_{0} \in \mathbb{R}$,

$$
\begin{gathered}
\left|f_{1}(t, y, u)-f_{1}\left(t, y, u_{0}\right)\right| \leq \sigma_{1}\left|u-u_{0}\right| \\
\left|f_{2}(t, x, v)-f_{2}\left(t, x, v_{0}\right)\right| \leq \sigma_{2}\left|v-v_{0}\right| .
\end{gathered}
$$

For convenience, set

$$
\begin{gathered}
\kappa_{1}:=\left(1+2 \sigma_{1}\right)^{-1}, \quad \kappa_{2}:=\left(1+2 \sigma_{2}\right)^{-1} \\
F_{1}(t, y, u):=u-f_{1}(t, y, u), \quad F_{2}(t, x, v):=v-f_{2}(t, x, v) .
\end{gathered}
$$
lemmas.

In order to convert system (1) into explicit form, we also need to list the following two

Lemma 2. Assume that Hypothesis 3 holds, Then, there exist two continuous functions $u(t)=$ $\varphi_{1}(t, y(t))$ and $v(t)=\varphi_{2}(t, x(t))$, satisfying

$$
\left\{\begin{array}{l}
F_{1}(t, y(t), u(t))=0,0<t<1 ; \\
F_{2}(t, x(t), v(t))=0,0<t<1 ，
\end{array}\right.
$$

for each pair of $(x, y) \in \mathcal{X} \times \mathcal{X}$.

Proof of Lemma 2. For each $y \in \mathcal{X}$, define a mapping $\mathcal{F}: \mathcal{X} \rightarrow \mathcal{X}$ by

$$
\mathcal{F} \varphi=\varphi-\kappa_{1} F_{1}(\cdot, y, \varphi) .
$$

Then, we claim that the mapping $\mathcal{F}$ is contractive.

In fact, by Hypothesis 3 , for each $\varphi, \varphi_{0} \in \mathcal{X}$, by (3), (4), and Hypothesis 3 , one can get that 


$$
\begin{aligned}
\left|\mathcal{F} \varphi(t)-\mathcal{F} \varphi_{0}(t)\right| & =\left|\varphi(t)-\varphi_{0}(t)-\kappa_{1}\left[F_{1}(t, y(t), \varphi(t))-F_{1}\left(t, y(t), \varphi_{0}(t)\right)\right]\right| \\
& =\left|\varphi(t)-\varphi_{0}(t)-\kappa_{1}\left[\varphi(t)-f_{1}(t, y(t), \varphi(t))-\varphi_{0}(t)+f_{1}\left(t, y(t), \varphi_{0}(t)\right)\right]\right| \\
& =\left|\left(1-\kappa_{1}\right)\left[\varphi(t)-\varphi_{0}(t)\right]-\kappa_{1}\left[f_{1}(t, y(t), \varphi(t))-f_{1}\left(t, y(t), \varphi_{0}(t)\right)\right]\right| \\
& \leq\left(1-\kappa_{1}\right)\left|\varphi(t)-\varphi_{0}(t)\right|+\sigma_{1} \kappa_{1}\left|\varphi(t)-\varphi_{0}(t)\right| \\
& =3 \sigma_{1} \cdot \kappa_{1}\left|\varphi(t)-\varphi_{0}(t)\right| .
\end{aligned}
$$

By (5), we have

$$
\left\|\mathcal{F} \varphi-\mathcal{F} \varphi_{0}\right\| \leq \varrho\left\|\varphi-\varphi_{0}\right\|
$$

where $\varrho:=3 \sigma_{1} \cdot \kappa_{1} \in(0,1)$. Namely, $\mathcal{F}$ is a contractive mapping in $\mathcal{X}$. Applying the contraction mapping principle, one can get that there exists continuous function $u(t)=$ $\varphi_{1}(t, y(t))$, satisfying $F_{1}(t, y(t), u(t))=0, t \in[0,1]$, for $y \in \mathcal{X}$.

From a process similar to the above, there exists the continuous function $v(t)=$ $\varphi_{2}(t, x(t))$ satisfying $F_{2}(t, x(t), v(t))=0, t \in[0,1]$, for $x \in \mathcal{X}$.

To sum up, the proof is complete.

Lemma 3. Define the mapping $G: \mathcal{X} \times \mathcal{X} \rightarrow \mathcal{X} \times \mathcal{X}$ by $G=\left(\varphi_{1}, \varphi_{2}\right)$. Assume that Hypothesis 1 and Hypothesis 3 hold. Then, the mapping $G$ is continuous.

Proof of Lemma 3. Let $\left(x_{n}, y_{n}\right) \rightarrow(x, y) \in \mathcal{X} \times \mathcal{X}$ as $n \rightarrow \infty$. For convenience, set $u_{n}:=\varphi_{1}\left(\cdot, y_{n}\right), v_{n}:=\varphi_{2}\left(\cdot, x_{n}\right)$. Then, we need only to claim that

$$
G\left(u_{n}, v_{n}\right) \rightarrow G(u, v), \text { as } \mathrm{n} \rightarrow \infty .
$$

To this end, by Hypothesis 1, Hypothesis 3, and Lemma 2, one can get that, for each $t \in[0,1]$

$$
\begin{aligned}
\left|u_{n}-u\right| & =\left|\varphi_{1}\left(t, y_{n}\right)-\varphi_{1}(t, y)\right| \\
& =\left|f_{1}\left(t, y_{n}, u_{n}\right)-f_{1}(t, y, u)\right| \\
& \leq\left|f_{1}\left(t, y_{n}, u_{n}\right)-f_{1}\left(t, y_{n}, u\right)\right|+\left|f_{1}\left(t, y_{n}, u\right)-f_{1}(t, y, u)\right| \\
& \leq \sigma_{1}\left|u_{n}-u\right|+\left|f_{1}\left(t, y_{n}, u\right)-f_{1}(t, y, u)\right|
\end{aligned}
$$

Taking the maximum for both sides of the above inequality in $t \in[0,1]$, we immediately get that

$$
\left\|u_{n}-u\right\| \leq \frac{1}{1-\sigma_{1}} \max _{t \in[0,1]}\left|f_{1}\left(t, y_{n}, u\right)-f_{1}(t, y, u)\right|
$$

So,

$$
\left\|u_{n}-u\right\| \rightarrow 0 \text { as } \mathrm{n} \rightarrow \infty
$$

Similarly,

$$
\left\|v_{n}-v\right\| \rightarrow 0 \text { as } \mathrm{n} \rightarrow \infty .
$$

As a result, the conclusion of this lemma follows.

Based on the Lemmas 2 and 3, we know that the existence of nontrivial solutions for problem (1) is equivalent to the existence of the following explicit fractional equations, as

$$
\left\{\begin{array}{l}
\mathcal{D}^{\alpha} x(t)=\varphi_{1}(t, y(t)), \quad 0<t<1 ; \\
\mathcal{D}^{\beta} y(t)=\varphi_{2}(t, x(t)), \quad 0<t<1 ; \\
x(0)=h_{1}(x), \quad x(1)=\int_{0}^{1} g_{1}(t) x(t) d t \\
y(0)=h_{2}(y), \quad y(1)=\int_{0}^{1} g_{2}(t) y(t) d t .
\end{array}\right.
$$

Therefore, we need only to investigate the existence of nontrivial solutions for (6). 
Lemma 4. Let $h \in \mathcal{X}$ be a given function. Then, the unique solution of the following boundary value problem

$$
\left\{\begin{array}{l}
\mathcal{D}^{\alpha} x(t)=h(t), \quad 0<t<1 \\
x(0)=h_{1}(x), \quad x(1)=\int_{0}^{1} g_{1}(t) x(t) d t
\end{array}\right.
$$

is given by

$$
x(t)=\left(1-\frac{\delta_{1}}{\delta_{2}}\right) h_{1}(x)+\int_{0}^{1} G_{1}(t, s) h(s) d s,
$$

where

$$
\begin{gathered}
G_{1}(t, s)=G_{1 \alpha}(t, s)+G_{2 \alpha}(t, s), \\
G_{1 \alpha}(t, s)=\left\{\begin{array}{c}
\frac{(t-s)^{\alpha-1}-t(1-s)^{\alpha-1}}{\Gamma(\alpha)}, \quad 0 \leq s \leq t \leq 1, \\
\frac{-t(1-s)^{\alpha-1}}{\Gamma(\alpha)}, \quad 0 \leq t \leq s \leq 1 ;
\end{array}\right. \\
G_{2 \alpha}(t, s)=\frac{t}{\delta_{2}} \int_{0}^{1} g_{1}(\tau) G_{1 \alpha}(\tau, s) d \tau .
\end{gathered}
$$

Proof of Lemma 4. By using Lemma 1, we can reduce (6) to the following equivalent equation

$$
\begin{aligned}
x(t) & =c_{0}+c_{1} t+\mathcal{I}^{\alpha} h(t) \\
& =c_{0}+c_{1} t+\frac{1}{\Gamma(\alpha)} \int_{0}^{t}(t-s)^{\alpha-1} h(s) d s,
\end{aligned}
$$

for some $c_{i} \in \mathbb{R}, \quad i=0,1$.

Applying boundary conditions to (9), one can obtain that

$$
c_{0}=h_{1}(x), \quad c_{1}=-h_{1}(x)-\frac{1}{\Gamma(\alpha)} \int_{0}^{1}(1-s)^{\alpha-1} h(s) d s+\int_{0}^{1} g_{1}(t) x(t) d t .
$$

Putting (10) into (9), we have

$$
\begin{aligned}
x(t) & =h_{1}(x)+\left[-h_{1}(x)-\frac{1}{\Gamma(\alpha)} \int_{0}^{1}(1-s)^{\alpha-1} h(s) d s+\int_{0}^{1} g_{1}(t) x(t) d t\right] \cdot t \\
& +\frac{1}{\Gamma(\alpha)} \int_{0}^{t}(t-s)^{\alpha-1} h(s) d s \\
& =(1-t) h_{1}(x)-\frac{t}{\Gamma(\alpha)} \int_{0}^{1}(1-s)^{\alpha-1} h(s) d s+\frac{1}{\Gamma(\alpha)} \int_{0}^{t}(t-s)^{\alpha-1} h(s) d s \\
& +t \int_{0}^{1} g_{1}(t) x(t) d t \\
& =(1-t) h_{1}(x)+\int_{0}^{t}\left[\frac{(t-s)^{\alpha-1}-t(1-s)^{\alpha-1}}{\Gamma(\alpha)}\right] h(s) d s-\int_{t}^{1} \frac{t(1-s)^{\alpha-1}}{\Gamma(\alpha)} h(s) d s \\
& +t \int_{0}^{1} g_{1}(\tau) x(\tau) d \tau \\
& =(1-t) h_{1}(x)+\int_{0}^{1} G_{1 \alpha}(t, s) h(s) d s+t \int_{0}^{1} g_{1}(\tau) x(\tau) d \tau .
\end{aligned}
$$

Moreover, by (11) one can derive

$$
\begin{aligned}
\int_{0}^{1} g_{1}(\tau) x(\tau) d \tau & =\int_{0}^{1} g_{1}(\tau)\left[(1-\tau) h_{1}(x)+\int_{0}^{1} G_{1 \alpha}(\tau, s) h(s) d s+\tau \int_{0}^{1} g_{1}(\tau) x(\tau) d \tau\right] d \tau \\
& =h_{1}(x) \cdot \int_{0}^{1}(1-\tau) g_{1}(\tau) d \tau+\int_{0}^{1} g_{1}(\tau) \cdot\left[\int_{0}^{1} G_{1 \alpha}(\tau, s) h(s) d s\right] d \tau \\
& +\left(\int_{0}^{1} g_{1}(\tau) x(\tau) d \tau\right) \cdot\left(\int_{0}^{1} g_{1}(\tau) \tau d \tau\right),
\end{aligned}
$$

which indicates that

$$
\int_{0}^{1} g_{1}(\tau) x(\tau) d \tau=\frac{1}{\delta_{2}} \cdot\left[h_{1}(x) \cdot \int_{0}^{1}(1-\tau) g_{1}(\tau) d \tau+\int_{0}^{1} g_{1}(\tau) \cdot\left[\int_{0}^{1} G_{1 \alpha}(\tau, s) h(s) d s\right] d \tau\right] .
$$


Substituting (13) into (11), we obtain

$$
\begin{aligned}
x(t) & =\left[1-t+\frac{t}{\delta_{2}} \int_{0}^{1}(1-\tau) g_{1}(\tau) d \tau\right] \cdot h_{1}(x)+\int_{0}^{1} G_{1 \alpha}(t, s) h(s) d s \\
& +\frac{t}{\delta_{2}} \int_{0}^{1} g_{1}(\tau) \int_{0}^{1} G_{1 \alpha}(\tau, s) h(s) d s d \tau \\
& =\left(1-\frac{\delta_{1}}{\delta_{2}} t\right) \cdot h_{1}(x)+\int_{0}^{1} G_{1 \alpha}(t, s) h(s) d s+\int_{0}^{1} G_{2 \alpha}(t, s) h(s) d s \\
& =\left(1-\frac{\delta_{1}}{\delta_{2}} t\right) \cdot h_{1}(x)+\int_{0}^{1} G_{1}(t, s) h(s) d s .
\end{aligned}
$$

This completes the proof.

From Lemmas 2-4, and Hypothesis 1, we need to only solve the following system of integral equations:

$$
\left\{\begin{array}{l}
x(t)=\left(1-\frac{\delta_{1}}{\delta_{2}} t\right) h_{1}(x)+\int_{0}^{1} G_{1}(t, s) \varphi_{1}(s, y(s)) d s \\
y(t)=\left(1-\frac{\gamma_{1}}{\gamma_{2}} t\right) h_{2}(y)+\int_{0}^{1} G_{2}(t, s) \varphi_{2}(s, x(s)) d s .
\end{array}\right.
$$

Now, define operators $\mathcal{A}=\left(\mathcal{A}_{1}, \mathcal{A}_{2}\right), \mathcal{B}=\left(\mathcal{B}_{1}, \mathcal{B}_{2}\right)$, and $\mathcal{T}=\mathcal{A}+\mathcal{B}$, where $\mathcal{A}_{1}, \mathcal{A}_{2}$ : $\mathcal{X} \rightarrow \mathcal{X}$ and $\mathcal{B}_{1}, \mathcal{B}_{2}: \mathcal{X} \times \mathcal{X} \rightarrow \mathcal{X} \times \mathcal{X}$ are defined by

$$
\begin{gathered}
\mathcal{A}_{1}(x)(t)=\left(1-\frac{\delta_{1}}{\delta_{2}} t\right) h_{1}(x), \quad \mathcal{A}_{2}(y)(t)=\left(1-\frac{\gamma_{1}}{\gamma_{2}} t\right) h_{2}(y) ; \\
\mathcal{B}_{1}(x, y)(t)=\int_{0}^{1} G_{1}(t, s) \varphi_{1}(s, y(s)) d s \\
\mathcal{B}_{2}(x, y)(t)=\int_{0}^{1} G_{2}(t, s) \varphi_{2}(s, x(s)) d s
\end{gathered}
$$

and

$$
\begin{gathered}
\mathcal{A}(x, y)=\left(\mathcal{A}_{1}(x), \mathcal{A}_{2}(y)\right), \\
\mathcal{B}(x, y)=\left(\mathcal{B}_{1}(x, y), \mathcal{B}_{2}(x, y)\right), \\
\mathcal{T}(x, y)=\mathcal{A}(x, y)+\mathcal{B}(x, y) .
\end{gathered}
$$

Then (15) in operator form becomes

$$
(u, v)=\mathcal{T}(u, v)
$$

Obviously, (16) guarantees that the existence of solutions for (15) is equivalent to the existence of the fixed point of $\mathcal{T}$ in $\mathcal{X} \times \mathcal{X}$. Therefore, we just need to find the fixed point of $\mathcal{T}$ in the following work.

\section{Existence Results}

In this section, we will present the existence result of solutions for problem (1). To do this, first we give some properties of mapping $\mathcal{T}$.

Lemma 5. Assume that Hypothesis 1 and Hypothesis 2 hold. Then, mapping $\mathcal{A}$ satisfies the Liptschitz condition. Namely, there exists $k \in(0,1)$, such that

$$
\left\|\mathcal{A}(x, y)-\mathcal{A}\left(x_{0}, y_{0}\right)\right\| \leq k\left\|(x, y)-\left(x_{0}, y_{0}\right)\right\|, \quad \forall(x, y),\left(x_{0}, y_{0}\right) \in \mathcal{X} \times \mathcal{X} .
$$

Consequently, it is a k-set contraction.

Proof of Lemma 5. For $(x, y),\left(x_{0}, y_{0}\right) \in \mathcal{X} \times \mathcal{X}$, by virtue of Hypothesis 2, we can obtain 


$$
\begin{aligned}
\left\|\mathcal{A}(x, y)-\mathcal{A}\left(x_{0}, y_{0}\right)\right\| & =\left\|\left(\mathcal{A}_{1}(x)-\mathcal{A}_{1}\left(x_{0}\right), \mathcal{A}_{2}(y)-\mathcal{A}_{2}\left(y_{0}\right)\right)\right\| \\
& =\left\|\mathcal{A}_{1}(x)-\mathcal{A}_{1}\left(x_{0}\right)\right\|+\left\|\mathcal{A}_{2}(y)-\mathcal{A}_{2}\left(y_{0}\right)\right\| \\
& =\max _{t \in[0,1]}\left|\left(1-\frac{\delta_{1}}{\delta_{2}} t\right)\left(h_{1}(x)-h_{1}\left(x_{0}\right)\right)\right|+\max _{t \in[0,1]}\left|\left(1-\frac{\gamma_{1}}{\gamma_{2}} t\right)\left(h_{2}(y)-h_{2}\left(y_{0}\right)\right)\right| \\
& \leq\left(1+\frac{\delta_{1}}{\delta_{2}}\right) k_{1} \max _{t \in[0,1]}\left|x-x_{0}\right|+\left(1+\frac{\gamma_{1}}{\gamma_{2}}\right) k_{2} \max _{t \in[0,1]}\left|y-y_{0}\right| \\
& =\left(1+\frac{\delta_{1}}{\delta_{2}}\right) k_{1}\left\|x-x_{0}\right\|+\left(1+\frac{\gamma_{1}}{\gamma_{2}}\right) k_{2}\left\|y-y_{0}\right\| \\
& \leq k\left(\left\|x-x_{0}\right\|+\left\|y-y_{0}\right\|\right) \\
& :=k\left\|(x, y)-\left(x_{0}, y_{0}\right)\right\|, \\
\text { where } k & =\max \left\{\left(1+\frac{\delta_{1}}{\delta_{2}}\right) k_{1},\left(1+\frac{\gamma_{1}}{\gamma_{2}}\right) k_{2}\right\}<1 .
\end{aligned}
$$

Therefore, mapping $\mathcal{A}$ satisfies the Liptschitz condition. By Proposition 1, we immediately get that mapping $\mathcal{A}$ is a $k$-set contraction.

Lemma 6. Assume that Hypothesis 1 and Hypothesis 3 hold. Then, mapping $\mathcal{B}: \mathcal{X} \times \mathcal{X} \rightarrow$ $\mathcal{X} \times \mathcal{X}$ is completely continuous.

Proof of Lemma 6. This proof will be carried out in two steps.

Claim 1. Mapping $\mathcal{B}$ is continuous.

In fact, for each $r>0$, set $U_{r}(0):=\{(x, y) \in \mathcal{X} \times \mathcal{X}:\|(x, y)\|<r\}$. Let $\left\{\left(x_{n}, y_{n}\right)\right\}_{n \in \mathbb{N}}$ be a sequence in $U_{r}(0)$, such that

$$
\left(x_{n}, y_{n}\right) \rightarrow\left(x_{0}, y_{0}\right) \text {, as } n \rightarrow+\infty \text {. }
$$

Now, we show

$$
\left\|\mathcal{B}\left(x_{n}, y_{n}\right)-\mathcal{B}\left(x_{0}, y_{0}\right)\right\| \rightarrow 0 \text {, as } n \rightarrow+\infty
$$

To this end, notice that, for each, $t \in[0,1]$,

$$
\begin{aligned}
\left|\mathcal{B}_{1}\left(x_{n}, y_{n}\right)(t)-\mathcal{B}_{1}\left(x_{0}, y_{0}\right)(t)\right| & =\left|\int_{0}^{1} G_{1}(t, s) \varphi_{1}\left(s, y_{n}(s)\right) d s-\int_{0}^{1} G_{1}(t, s) \varphi_{1}\left(s, y_{0}(s)\right) d s\right| \\
& =\left|\int_{0}^{1} G_{1}(t, s)\left[\varphi_{1}\left(s, y_{n}(s)\right)-\varphi_{1}\left(s, y_{0}(s)\right)\right] d s\right| \\
& \leq\left(\max _{s \in[0,1]}\left|G_{1}(t, s)\right|\right) \cdot\left(\int_{0}^{1}\left|\varphi_{1}\left(s, y_{n}(s)\right)-\varphi_{1}\left(s, y_{0}(s)\right)\right| d s\right) .
\end{aligned}
$$

Taking the maximum for both sides of the above inequality in $t \in[0,1]$, one can find that

$$
\left\|\mathcal{B}_{1}\left(x_{n}, y_{n}\right)-\mathcal{B}_{1}\left(x_{0}, y_{0}\right)\right\| \leq\left(\max _{t, s \in[0,1]}\left|G_{1}(t, s)\right|\right) \cdot\left(\int_{0}^{1}\left|\varphi_{1}\left(s, y_{n}(s)\right)-\varphi_{1}\left(s, y_{0}(s)\right)\right| d s\right) .
$$

This, together with Lemmas 2 and 3, implies that

$$
\left\|\mathcal{B}_{1}\left(x_{n}, y_{n}\right)-\mathcal{B}_{1}\left(x_{0}, y_{0}\right)\right\| \rightarrow 0 \text {, as } n \rightarrow+\infty \text {. }
$$

Similarly,

$$
\left\|\mathcal{B}_{2}\left(x_{n}, y_{n}\right)-\mathcal{B}_{2}\left(x_{0}, y_{0}\right)\right\| \rightarrow 0 \text {, as } n \rightarrow+\infty \text {. }
$$

Claim 2. Mapping $\mathcal{B}$ is compact.

To this end, let $\left\{\left(u_{n}, v_{n}\right)\right\}_{n \in \mathbb{N}}$ be a sequence in $U_{r}(0)$. Then, one can see that $\mathcal{B}\left(\left\{\left(u_{n}, v_{n}\right)\right\}\right.$ $n \in \mathbb{N})$ is bounded. So, we need to only show that $\mathcal{B}\left(\left\{\left(u_{n}, v_{n}\right)\right\}_{n \in \mathbb{N}}\right)$ is equicontinuous. 
In fact, the definition of $\left\{\left(u_{n}, v_{n}\right)\right\}_{n \in \mathbb{N}}$, together with Lemma 3, guarantees that there exists $M_{1}>0$ such that

$$
\mid \varphi_{1}\left(t, v_{n}(t) \mid \leq M_{1}, \forall n \in \mathbb{N}, t \in[0,1] .\right.
$$

Thus, for $t, \ell \in[0,1]$, we can have that

$$
\begin{aligned}
\left|\mathcal{B}_{1}\left(u_{n}, v_{n}\right)(t)-\mathcal{B}_{1}\left(u_{n}, v_{n}\right)(\ell)\right| & =\left|\int_{0}^{1} G_{1}(t, s) \varphi_{1}\left(s, v_{n}(s)\right) d s-\int_{0}^{1} G_{1}(\ell, s) \varphi_{1}\left(s, v_{n}(s)\right) d s\right| \\
& \leq \int_{0}^{1}\left|G_{1}(t, s)-G_{1}(\ell, s)\right| \cdot\left|\varphi_{1}\left(s, v_{n}(s)\right)\right| d s \\
& \leq M_{1} \int_{0}^{1}\left|G_{1}(t, s)-G_{1}(\ell, s)\right| d s .
\end{aligned}
$$

Thus, $\mathcal{B}_{1}\left(\left\{\left(u_{n}, v_{n}\right)\right\}_{n \in \mathbb{N}}\right)$ is equicontinuous. Similarly, $\mathcal{B}_{2}\left(\left\{\left(u_{n}, v_{n}\right)\right\}_{n \in \mathbb{N}}\right)$ is equicontinuous. As a result, $\mathcal{B}(E)$ is equicontinuous. Therefore, by Arzela-Ascoli theorem, $\mathcal{B}(E)$ is compact.

To sum up, the conclusion of this lemma follows.

Now we are in a position to prove our existence result of solutions in the following.

Theorem 2. Assume that Hypotheses 1-3 hold. Then problem (1) has at least one solution in $\mathcal{X} \times \mathcal{X}$

Proof of Theorem 2. From Lemmas 5 and 6 , we know that $\mathcal{A}$ is a $k$-set contraction and $\mathcal{B}$ is a 0 -set contraction, respectively. Thus, Definition 3 and Proposition 3 indicate that $\mathcal{T}$ is condensing. Define

$$
\Lambda:=\{(x, y) \in \mathcal{X} \times \mathcal{X}: \exists \xi \in[0,1] \text { with }(x, y)=\xi \mathcal{T}(x, y)\} \subset \mathcal{X} \times \mathcal{X} .
$$

Now, we claim that $\Lambda$ is bounded in $\mathcal{X} \times \mathcal{X}$.

To this end, by Hypothesis 2 , one can get that for $(x, y) \in \Lambda$,

$$
\begin{aligned}
\|\mathcal{A}(x, y)\| & =\left\|\mathcal{A}_{1}(x)\right\|+\left\|\mathcal{A}_{2}(y)\right\| \\
& =\max _{t \in[0,1]}\left|\left(1-\frac{\delta_{1}}{\delta_{2}} t\right) h_{1}(x)\right|+\max _{t \in[0,1]}\left|\left(1-\frac{\gamma_{1}}{\gamma_{2}} t\right) h_{2}(y)\right| \\
& \leq\left(1+\frac{\delta_{1}}{\delta_{2}}\right)\left(\left\|h_{1}(0)\right\|+k_{1}\|x\|\right)+\left(1+\frac{\gamma_{1}}{\gamma_{2}}\right)\left(\left\|h_{2}(0)\right\|+k_{2}\|y\|\right) \\
& =\left\{\left(1+\frac{\delta_{1}}{\delta_{2}}\right)\left\|h_{1}(0)\right\|+\left(1+\frac{\gamma_{1}}{\gamma_{2}}\right)\left\|h_{2}(0)\right\|\right\}+k\|(x, y)\| \\
& :=\mathrm{Y}_{a}+k\|(x, y)\|,
\end{aligned}
$$

where $Y_{a}=\left(1+\frac{\delta_{1}}{\delta_{2}}\right)\left\|h_{1}(0)\right\|+\left(1+\frac{\gamma_{1}}{\gamma_{2}}\right)\left\|h_{2}(0)\right\|$ and

$k=\max \left\{\left(1+\frac{\delta_{1}}{\delta_{2}}\right) k_{1},\left(1+\frac{\gamma_{1}}{\gamma_{2}}\right) k_{2}\right\}<1$.

$$
\begin{aligned}
\|\mathcal{B}(x, y)\| & =\left\|\mathcal{B}_{1}(x, y)\right\|+\left\|\mathcal{B}_{2}(x, y)\right\| \\
& =\left|\int_{0}^{1} G_{1}(t, s) \varphi_{1}(s, y(s)) d s\right|+\left|\int_{0}^{1} G_{2}(t, s) \varphi_{2}(s, x(s)) d s\right| \\
& \leq\left(\int_{0}^{1} \max _{t \in[0,1]}\left|G_{1}(t, s)\right| d s+\int_{0}^{1}\left|\varphi_{1}(s, y(s))\right| d s\right) \\
& +\left(\int_{0}^{1} \max _{t \in[0,1]}\left|G_{2}(t, s)\right| d s+\int_{0}^{1}\left|\varphi_{2}(s, x(s))\right| d s\right) \\
& :=Y_{b} .
\end{aligned}
$$


From (19) and (20), it follows that

$$
\|(x, y)\| \leq\|\mathcal{T}(x, y)\|=\|\mathcal{A}(x, y)\|+\|\mathcal{B}(x, y)\| \leq \mathrm{Y}_{a}+\mathrm{Y}_{b}+k\|(x, y)\| .
$$

Thus,

$$
\|(x, y)\| \leq \frac{\mathrm{Y}_{a}+\mathrm{Y}_{b}}{1-k} .
$$

Then, $\Lambda$ is bounded in $\mathcal{X} \times \mathcal{X}$. Hence, by Theorem $1, \mathcal{T}$ has at least one fixed point. The conclusion of this theorem follows.

\section{Stable Results}

In this section, we investigate the Ulam Stability for problem (1). First, let us list the following assumption.

Hypothesis 4. There exist constants $c_{\alpha}, c_{\beta} \in(0,+\infty)$ and $d_{\alpha}, d_{\beta} \in(0,1)$, such that

$$
\begin{aligned}
& \left|f_{1}(t, y, u)-f_{1}\left(t, y_{0}, u_{0}\right)\right| \leq c_{\alpha}\left|y-y_{0}\right|+d_{\alpha}\left|u-u_{0}\right|, \forall y, y_{0}, u, u_{0} \in \mathbb{R}, \\
& \left|f_{2}(t, x, v)-f_{2}\left(t, x_{0}, v_{0}\right)\right| \leq c_{\beta}\left|x-x_{0}\right|+d_{\beta}\left|u-u_{0}\right|, \forall x, x_{0}, v, v_{0} \in \mathbb{R} .
\end{aligned}
$$

Next, for some $\varepsilon=\left(\varepsilon_{1}, \varepsilon_{2}\right)>0$, consider the following differential inequalities:

$$
\left\{\begin{array}{l}
\left|\mathcal{D}^{\alpha} x(t)-f_{1}\left(t, y(t), \mathcal{D}^{\alpha} x(t)\right)\right| \leq \varepsilon_{1}, 0<t<1, \\
\left|\mathcal{D}^{\beta} y(t)-f_{2}\left(t, x(t), \mathcal{D}^{\beta} y(t)\right)\right| \leq \varepsilon_{2}, 0<t<1, \\
x(0)=h_{1}(x), \quad x(1)=\int_{0}^{1} g_{1}(t) x(t) d t, \\
y(0)=h_{2}(y), y(1)=\int_{0}^{1} g_{2}(t) y(t) d t .
\end{array}\right.
$$

Definition 4. ([40]) Problem (1) is Ulam-Hyers stable, if there exists $L_{(1,2)}=\left(L_{1}, L_{2}\right)>0$, such that for each solution $(x, y) \in \mathcal{X} \times \mathcal{X}$ of (21), there is a unique solution $(\bar{x}, \bar{y}) \in \mathcal{X} \times \mathcal{X}$ of (1) with

$$
|(x, y)(t)-(\bar{x}, \bar{y})(t)| \leq L_{(1,2)} \varepsilon, \quad t \in[0,1] .
$$

Definition 5. ([40]) Problem (1) is generalized Ulam-Hyers stable, if there exists $\Phi_{(1,2)} \in$ $C\left(\mathbb{R}^{+}, \mathbb{R}^{+}\right)$with $\Phi_{(1,2)}(0)=0$, such that for each solution $(x, y) \in \mathcal{X} \times \mathcal{X}$ of $(21)$, there is a unique solution $(\bar{x}, \bar{y}) \in \mathcal{X} \times \mathcal{X}$ of (1) with

$$
|(x, y)(t)-(\bar{x}, \bar{y})(t)| \leq \Phi_{(1,2)}(\varepsilon), \quad t \in[0,1] .
$$

Moreover, we also need the following lemma in stability analysis.

Lemma 7. Let $(x, y) \in \mathcal{X} \times \mathcal{X}$ be a solution of inequalities (21). Then, the following inequalities hold:

$$
\left\{\begin{array}{l}
\left|x(t)-\left(1-\frac{\delta_{1}}{\delta_{2}} t\right) h_{1}(x)-\int_{0}^{1} G_{1}(t, s) f_{1}\left(s, y(s), \mathcal{D}^{\alpha} x(s)\right) d s\right| \leq L_{1} \varepsilon_{1} \\
\left|y(t)-\left(1-\frac{\gamma_{1}}{\gamma_{2}} t\right) h_{2}(y)-\int_{0}^{1} G_{2}(t, s) f_{2}\left(s, x(s), \mathcal{D}^{\beta} y(s)\right) d s\right| \leq L_{2} \varepsilon_{2}
\end{array}\right.
$$

where $L_{1}=\max _{t \in[0,1]} \int_{0}^{1}\left|G_{1}(t, s)\right| d s, \quad L_{2}=\max _{t \in[0,1]} \int_{0}^{1}\left|G_{2}(t, s)\right| d s$.

Proof of Lemma 7. Obviously, there exist $\Phi_{1}, \Phi_{2} \in \mathcal{X}$, which depend on $x, y$, respectively, such that $\left|\Phi_{1}(t)\right| \leq \varepsilon_{1},\left|\Phi_{2}(t)\right| \leq \varepsilon_{2}$; 
and

$$
\left\{\begin{array}{l}
\mathcal{D}^{\alpha} x(t)=f_{1}\left(t, y(t), \mathcal{D}^{\alpha} x(t)\right)+\Phi_{1}(t), 0<t<1 \\
\mathcal{D}^{\beta} y(t)=f_{2}\left(t, x(t), \mathcal{D}^{\beta} y(t)\right)+\Phi_{2}(t), 0<t<1 \\
x(0)=h_{1}(x), x(1)=\int_{0}^{1} g_{1}(t) x(t) d t \\
y(0)=h_{2}(y), y(1)=\int_{0}^{1} g_{2}(t) y(t) d t
\end{array}\right.
$$

By using Lemma 4, one can see that

$$
\left\{\begin{array}{l}
x(t)=\left(1-\frac{\delta_{1}}{\delta_{2}} t\right) h_{1}(x)+\int_{0}^{1} G_{1}(t, s) f_{1}\left(s, y(s), \mathcal{D}^{\alpha} x(s)\right) d s+\int_{0}^{1} G_{1}(t, s) \Phi_{1}(s) d s, \\
y(t)=\left(1-\frac{\gamma_{1}}{\gamma_{2}} t\right) h_{2}(y)+\int_{0}^{1} G_{2}(t, s) f_{2}\left(s, x(s), \mathcal{D}^{\beta} y(s)\right) d s+\int_{0}^{1} G_{2}(t, s) \Phi_{2}(s) d s .
\end{array}\right.
$$

From (23), we have

$$
\begin{aligned}
& \left|x(t)-\left(1-\frac{\delta_{1}}{\delta_{2}} t\right) h_{1}(x)-\int_{0}^{1} G_{1}(t, s) f_{1}\left(s, y(s), \mathcal{D}^{\alpha} x(s)\right) d s\right| \\
& =\left|\int_{0}^{1} G_{1}(t, s) \Phi_{1}(s) d s\right| \\
& \leq \max _{t \in[0,1]} \int_{0}^{1}\left|G_{1}(t, s) \Phi_{1}(s)\right| d s \leq L_{1} \varepsilon_{1} .
\end{aligned}
$$

Similarly,

$$
\left|y(t)-\left(1-\frac{\gamma_{1}}{\gamma_{2}} t\right) h_{2}(y)-\int_{0}^{1} G_{2}(t, s) f_{2}\left(s, x(s), \mathcal{D}^{\beta} y(s)\right) d s\right| \leq L_{2} \varepsilon_{2} .
$$

The proof is complete.

Now, we are in a position to prove the main stable theorem of this section.

Theorem 3. Assume that Hypothesis 1 and $\left(H 3^{\prime}\right)$ hold. Then the solution of problem (1) is Ulam-Hyers stable if

$$
\Delta=1-\frac{L_{1} L_{2} c_{\alpha} c_{\beta}}{\left(1-d_{\alpha}\right)\left(1-d_{\beta}\right)}>0 .
$$

Furthermore, the solution of problem (1) is generalized Ulam-Hyers stable.

Proof of Lemma 3. Let $(x, y) \in \mathcal{X} \times \mathcal{X}$ be a solution of (21) and $(\bar{x}, \bar{y}) \in \mathcal{X} \times \mathcal{X}$ be the unique solution to (1). Then, in view of Lemma 4 , we have that

$$
\left\{\begin{array}{l}
\bar{x}(t)=\left(1-\frac{\delta_{1}}{\delta_{2}} t\right) h_{1}(\bar{x})+\int_{0}^{1} G_{1}(t, s) f_{1}\left(s, \bar{y}(s), \mathcal{D}^{\alpha} \bar{x}(s)\right) d s, \\
\bar{y}(t)=\left(1-\frac{\gamma_{1}}{\gamma_{2}} t\right) h_{2}(\bar{y})+\int_{0}^{1} G_{2}(t, s) f_{2}\left(s, \bar{x}(s), \mathcal{D}^{\beta} \bar{y}(s)\right) d s .
\end{array}\right.
$$

Now, consider

$$
\begin{aligned}
|x(t)-\bar{x}(t)| & =\left|x(t)-\left(1-\frac{\delta_{1}}{\delta_{2}} t\right) h_{1}(\bar{x})-\int_{0}^{1} G_{1}(t, s) f_{1}\left(s, \bar{y}(s), \mathcal{D}^{\alpha} \bar{x}(s)\right) d s\right| \\
& \leq\left|x(t)-\left(1-\frac{\delta_{1}}{\delta_{2}} t\right) h_{1}(\bar{x})-\int_{0}^{1} G_{1}(t, s) f_{1}\left(s, y(s), \mathcal{D}^{\alpha} x(s)\right) d s\right| \\
& +\left|\int_{0}^{1} G_{1}(t, s) f_{1}\left(s, y(s), \mathcal{D}^{\alpha} x(s)\right) d s-\int_{0}^{1} G_{1}(t, s) f_{1}\left(s, \bar{y}(s), \mathcal{D}^{\alpha} \bar{x}(s)\right) d s\right| \\
& \leq L_{1} \varepsilon_{1}+\left|\int_{0}^{1} G_{1}(t, s)\left(f_{1}\left(s, y(s), \mathcal{D}^{\alpha} x(s)\right)-f_{1}\left(s, \bar{y}(s), \mathcal{D}^{\alpha} \bar{x}(s)\right)\right)\right| .
\end{aligned}
$$


By $\left(\mathrm{H}^{\prime}\right)$, we have

$$
|x(t)-\bar{x}(t)| \leq L_{1} \varepsilon_{1}+\max _{t \in[0,1]} \int_{0}^{1}\left|G_{1}(t, s)\right| \cdot\left(\frac{c_{\alpha}}{1-d_{\alpha}}|y(s)-\bar{y}(s)|\right) d s .
$$

Thus,

$$
\|x-\bar{x}\| \leq L_{1} \varepsilon_{1}+\frac{L_{1} c_{\alpha}}{1-d_{\alpha}}\|y-\bar{y}\| .
$$

Similarly, we also have

$$
\|y-\bar{y}\| \leq L_{2} \varepsilon_{2}+\frac{L_{2} c_{\beta}}{1-d_{\beta}}\|x-\bar{x}\| .
$$

From (27) and (28), it follows that

$$
\left\{\begin{array}{c}
\|x-\bar{x}\|-\frac{L_{1} c_{\alpha}}{1-d_{\alpha}}\|y-\bar{y}\| \leq L_{1} \varepsilon_{1} \\
\|y-\bar{y}\|-\frac{L_{2} c_{\beta}}{1-d_{\beta}}\|x-\bar{x}\| \leq L_{2} \varepsilon_{2} .
\end{array}\right.
$$

We write (29) in matrix form as

$$
\left[\begin{array}{cc}
1 & -\frac{L_{1} c_{\alpha}}{1-d_{\alpha}} \\
-\frac{L_{2} c_{\beta}}{1-d_{\beta}} & 1
\end{array}\right] \cdot\left[\begin{array}{l}
\|x-\bar{x}\| \\
\|y-\bar{y}\|
\end{array}\right]=\left[\begin{array}{c}
L_{1} \varepsilon_{1} \\
L_{2} \varepsilon_{2}
\end{array}\right] .
$$

Then, we get

$$
\left[\begin{array}{l}
\|x-\bar{x}\| \\
\|y-\bar{y}\|
\end{array}\right] \leq\left[\begin{array}{cc}
\frac{1}{\Omega} & \frac{L_{1} c_{\beta}}{\Omega\left(1-d_{\beta}\right)} \\
\frac{L_{2} c_{\alpha}}{\Omega\left(1-d_{\alpha}\right)} & \frac{1}{\Omega}
\end{array}\right] \cdot\left[\begin{array}{c}
L_{1} \varepsilon_{1} \\
L_{2} \varepsilon_{2}
\end{array}\right],
$$

where $\Omega=1-\frac{L_{1} L_{2} c_{\alpha} c_{\beta}}{\left(1-d_{\alpha}\right)\left(1-d_{\beta}\right)} \neq 0$.

Further simplification gives

$$
\begin{aligned}
& \|x-\bar{x}\| \leq \frac{L_{1} \varepsilon_{1}}{\Omega}+\frac{L_{1} L_{2} c_{\beta} \varepsilon_{2}}{\Omega\left(1-d_{\beta}\right)}, \\
& \|y-\bar{y}\| \leq \frac{L_{2} \varepsilon_{2}}{\Omega}+\frac{L_{1} L_{2} c_{\alpha} \varepsilon_{1}}{\Omega\left(1-d_{\alpha}\right)} .
\end{aligned}
$$

From above, we have

$$
\|x-\bar{x}\|+\|y-\bar{y}\| \leq \frac{L_{1} \varepsilon_{1}}{\Omega}+\frac{L_{1} L_{2} c_{\beta} \varepsilon_{2}}{\Omega\left(1-d_{\beta}\right)}+\frac{L_{2} \varepsilon_{2}}{\Omega}+\frac{L_{1} L_{2} c_{\alpha} \varepsilon_{1}}{\Omega\left(1-d_{\alpha}\right)} .
$$

Taking $\varepsilon=\max \left\{\varepsilon_{1}, \varepsilon_{2}\right\}$, one can get that

$$
\|(x, y)-(\bar{x}, \bar{y})\| \leq L_{(1,2)} \varepsilon,
$$

where

$$
L_{(1,2)}=\frac{L_{1} \varepsilon_{1}}{\Omega}+\frac{L_{1} L_{2} c_{\beta} \varepsilon_{2}}{\Omega\left(1-d_{\beta}\right)}+\frac{L_{2} \varepsilon_{2}}{\Omega}+\frac{L_{1} L_{2} c_{\alpha} \varepsilon_{1}}{\Omega\left(1-d_{\alpha}\right)} .
$$

Therefore, the solution of system (1) is Ulam-Hyers stable. 
In addition, set $\Phi_{(1,2)}(\varepsilon)=L_{(1,2)} \varepsilon$, then $\Phi_{(1,2)}(0)=0$. By Definition 5, the problem (1) is generalized Ulam-Hyers stable.

\section{Illustrative Example}

Here, we give an example to show the effectiveness of the obtained results. Let us consider the following implicit-type fractional differential coupled system:

$$
\left\{\begin{array}{l}
\mathcal{D}^{\frac{3}{2}} x(t)=t+\sin (y(t))+\frac{1}{2} \mathcal{D}^{\frac{3}{2}} x(t), \quad 0<t<1 ; \\
\mathcal{D}^{\frac{5}{4}} y(t)=t+x(t)+\frac{1}{3} \mathcal{D}^{\frac{5}{4}} y(t), \quad 0<t<1 ; \\
x(0)=\frac{x}{8-2 e^{\prime}}, \quad x(1)=\int_{0}^{1} \frac{e^{t}}{2} x(t) d t \\
y(0)=\frac{y}{3}, \quad y(1)=\int_{0}^{1} e^{-t} y(t) d t .
\end{array}\right.
$$

System (33) can be regarded as a system of the form (1). Here,

$$
\begin{gathered}
\alpha=\frac{3}{2}, h_{1}(x)=\frac{x}{8-2 e}, \quad g_{1}(t)=\frac{e^{t}}{2} ; \\
\beta=\frac{5}{4}, h_{2}(y)=\frac{y}{3}, g_{2}(t)=e^{-t} ; \\
f_{1}\left(t, y(t), \mathcal{D}^{\alpha} x(t)\right)=t+\sin (y(t))+\frac{1}{2} \mathcal{D}^{\frac{3}{2}} x(t) ; \\
f_{2}\left(t, x(t), \mathcal{D}^{\beta} y(t)\right)=t+x(t)+\frac{1}{3} \mathcal{D}^{\beta} y(t) .
\end{gathered}
$$

By careful calculation and (2), one can obtain that

$$
\begin{aligned}
& \delta_{1}=\frac{3-e}{2}, \delta_{2}=\frac{1}{2}, \delta=\frac{1}{4-e} ; \\
& \gamma_{1}=e^{-1}, \quad \gamma_{2}=2 e^{-1}, \quad \gamma=\frac{2}{3} .
\end{aligned}
$$

Obviously, we can see that Hypothesis 1 holds. Now assumptions Hypothesis 2-(H3') are satisfied if we set

$$
k_{1}=\frac{1}{8-2 e}, k_{2}=\frac{1}{3}, \sigma_{1}=\frac{1}{2}, \sigma_{2}=\frac{1}{3}, c_{\alpha}=c_{\beta}=1, d_{\alpha}=\frac{1}{2}, d_{\beta}=\frac{1}{3} .
$$

Consequently, system (33) has at least one solution by Theorem 2 and the solution of system (33) is Ulam-Hyers stable and generalized Ulam-Hyers stable by Theorem 3.

\section{Discussion}

In this paper,firstly by means of topological degree theory, some existence results are obtained for a class of implicit-type coupled systems with integral boundary conditions, involving Caputo fractional derivatives. As far as we know, there are no existence results available for considered systems (1). Furthermore, the nonlinear terms here need only to satisfy the Lipschitz condition for one variable, which relax the restriction of the nonlinear terms in the literatures. The main reason is that the contraction mapping principle is used to obtain the existence of implicit function (see Lemmas 2 and 3). Secondly, UlamHyers stability and generalized Ulam-Hyers stability are investigated under some suitable assumptions. The method used in present paper can be applied in broadest context, such as many implicit-type systems with various boundary conditions involving integer or fractional derivatives. 
Author Contributions: Conceptualization and visualization, Y.L.; formal analysis and investigation, D.Z.; writing original draft and investigation, L.L. All authors have read and agreed to the published version of the manuscript.

Funding: This research was funded by NNSF of P.R.China (62073202), Natural Science Foundation of Shandong Province (ZR2020MA007), and a project of Shandong Province Higher Educational Science and Technology Program of China under the grant J18KA233.

Institutional Review Board Statement: Not applicable.

Informed Consent Statement: Not applicable.

Data Availability Statement: Not applicable.

Acknowledgments: The authors are thankful to the editor and anonymous referees for their valuable comments and suggestions.

Conflicts of Interest: The authors declare no conflict of interest.

\section{References}

1. Grigorenko, I.; Grigorenko, E. Chaotic dynamics of the fractional Lorenz system. Phys. Rev. Lett. 2003, 91, 034101. [CrossRef]

2. Ge, Z.M.; Jhuang, W.R. Chaos, control and synchronization of a fractional order rotational mechanical system with a centrifugal governor. Chaos Soliton Fract. 2007, 33, 270-289. [CrossRef]

3. Hartley, T.T.; Lorenzo, C.F.; Qammer, H.K. Chaos in a fractional order Chua's system. IEEE. Trans. 1995, 42, 485-490. [CrossRef]

4. Liu, Y. Bifurcation techniques for a class of boundary value problems of fractional impulsive differential equations. J. Nonlinear Sci. Appl. 2015, 8, 340-353. [CrossRef]

5. Wang, Y.; Liu, Y.; Cui, Y. Infinitely many solutions for impulsive fractional boundary value problem with $p$-Laplacian. Bound. Value Probl. 2018, 2018, 94. [CrossRef]

6. Kusnezov, D.; Bulgac, A.; Dang, G.D. Quantum Levy processes and fractional kinetics. Phys. Rev. Lett. 1999, 82, 1136-1139. [CrossRef]

7. Li, Y.; Chen, Y.Q.; Podlubny, I. Stability of fractional-order nonlinear dynamic systems: Lyapunov direct method and generalized Mittag-Leffler stability. Comput. Math. Appl. 2010, 59, 1810-1821. [CrossRef]

8. Zhao, D.; Liu, Y.; Li, X. Controllability for a class of semilinear fractional evolution systems via resolvent operators. Commun. Pure Appl. Anal. 2019, 18, 455-478. [CrossRef]

9. Cheng, H.; Yuan, R. The stability of the equilibria of the Allen-Cahn equation with fractional diffusion. Appl. Anal. 2019, 98 600-610. [CrossRef]

10. Jia, J.; Wang, H. A fast finite volume method for conservative space-time fractional diffusion equations discretized on space-time locally refined meshes. Comput. Math. Appl. 2019, 78, 1345-1356. [CrossRef]

11. Metzler, R.; Klafter, J. The random walks guide to anomalous diffusion: A fractional dynamics approach. Phys. Rep. 2000, 339 1-77. [CrossRef]

12. Zhou, Z.; Gong, W. Finite element approximation of optimal control problems governed by time fractional diffusion equation. Comput. Math. Appl. 2016, 71, 301-318. [CrossRef]

13. Ostoja-Starzewski, M. Towards thermoelasticity of fractal media. J. Therm. Stress 2007, 30, 889-896. [CrossRef]

14. Povstenko, Y. Fractional Thermoelasticity; Springer: New York, NY, USA, 2015.

15. Kilbas, A.A.A.; Srivastava, H.M.; Trujillo, J.J. Theory and Applications of Fractional Differential Equations; North-Holland Mathematics Studies 204; Elsevier Science B. V.: Amsterdam, The Netherlands, 2006.

16. Lakshmikantham, V.; Leela, S.; Devi, J.V. Theory of Fractional Dynamic Systems; Cambridge Scientific Publishers: Cambridge, UK, 2009.

17. Podlubny, I. Fractional Differential Equations, Mathematics in Science and Engineering; Academic Press: New York, NY, USA, 1999.

18. Samko, S.G.; Kilbas, A.A.; Marichev, O.I. Fractional Integral And Derivatives (Theory and Applications); Gordon and Breach: Montreux, Switzerland, 1993.

19. Ulam, S.M. Problems in Modern Mathematics; Science Editors: New York, NY, USA, 1940.

20. Hyers, D.H. On the stability of the linear functional equation. Proc. Natl. Acad. Sci. USA 1941, 27, 222-224. [CrossRef]

21. Murali, R.; Selvan, A.P. On the generlized Hyers-Ulam stability of linear ordinary differential equations of higher order. Int. J. Pure Appl. Math. 2017, 117, 317-326.

22. Rus, I.A. Ulam stability of ordinary differential equations. Stud. Univ. Babeş Bolyai Math. 2009, LIV, 125-133.

23. Dimplekumar, C.; Avadhesh, K. Existence, uniqueness and Ulam's stability of solutions for a coupled system of fractional differential equations with integral boundary conditions. Mathematics 2018, 6, 96.

24. Benchohra, M.; Lazreg, J.E. Existence and Ulam stability for nonlinear implicit fractional differential equations with Hadamard derivative. Stud. Univ. Babeş Bolyai Math. 2017, 62, 27-38. [CrossRef]

25. Ali, Z.; Zada, A.; Shah, K. Ulam stability to a toppled systems of nonlinear implicit fractional order boundary value problem. Bound. Value Probl. 2018, 2018, 175. [CrossRef] 
26. Khan, A.; Shah, K.; Li, Y.; Khan, T.S. Ulam type stability for a coupled systems of boundary value problems of nonlinear fractional differential equations. J. Funct. Space 2017, 2017, 3046013. [CrossRef]

27. Ahmad, B.; Alsaedi, A.; Alghamdi, B.S. Analytic approximation of solutions of the forced Duffing equation with integral boundary conditions. Nonlinear Anal. Real. 2008, 9, 1727-1740. [CrossRef]

28. Wang, Y.; Liu, L.; Zhang, X.; Wu, Y. Positive solutions of an abstract fractional semipositone differential system model for bioprocesses of HIV infection. Appl. Math. Comput. 2015, 258, 312-324. [CrossRef]

29. Čiegis, R.; Bugajev, A. Numerical approximation of one model of the bacterial self-organization. Nonlinear Anal. Model. 2012, 17 253-270. [CrossRef]

30. Ahmad, B.; Ntouyas, S.K.; Alsaedi, A. On a coupled system of fractional differential equations with coupled nonlocal and integral boundary conditions. Chaos Soliton. Fract. 2016, 83, 234-241. [CrossRef]

31. Mao, J.; Zhao, D. Multiple positive solutions for nonlinear fractional differential equations with integral boundary value conditions and a parameter. J. Funct. Space 2019, 2019, 2787569. [CrossRef]

32. Zhao, D.; Liu, Y. Positive solutions for a class of fractional differential coupled system with integral boundary value conditions. J. Nonlinear Sci. Appl. 2016, 9, 2922-2942. [CrossRef]

33. Zhao, D.; Liu, Y. Twin solutions to semipositone boundary value problems for fractional differential equations with coupled integral boundary conditions. J. Nonlinear Sci. Appl. 2017, 10, 3544-3565. [CrossRef]

34. Benchohra, M.; Lazreg, J.E. Nonlinear fractional implicit differential equations. Commun. Appl. Anal. $2013,17,471-482$.

35. Benchohra, M.; Lazreg, J.E. Existence and uniqueness results for nonlinear implicit fractional differential equations with boundary conditions. Rom. J. Math. Comput. Sci. 2014, 4, 60-72.

36. Benchohra, M.; Lazreg, J.E. On stability for nonlinear implicit fractional differential equations. Matematiche (Catania) 2015, 70, 49-61.

37. Nieto, J.J.; Ouahab, A.; Venktesh, V. Implicit fractional differential equations via the Liouville-Caputo derivative. Mathematics 2015, 3, 398-411. [CrossRef]

38. Samina KShah RA Khan, D. Baleanu, Study of implicit type coupled system of non-integer order differential equations with antiperiodic boundary conditions, Math. Meth. Appl. Sci. 2019, 42, 2033-2042. [CrossRef]

39. Guo, D.; Lakshmikantham, V. Nonlinear Problem in Abstract Cones; Academic Press: San Diego, CA, USA, 1988.

40. Ioan, A.R. Ulam stabilities of ordinary differential equations in a Banach space. Carpathian J. Math. 2010, 26, 103-107. 